\title{
UNITARY REPRESENTATIONS OF SOME LINEAR GROUPS II
}

\author{
SEIZÔ ITÔ
}

§ 0. Introudction. In his preceding paper [2], the author determined the types of irreducible unitary representations and cyclic unitary representations of the group of all euclidean motions in 2-space $E^{2}$. The purpose of the present paper is to determine the types of irreducible unitary representations and cyclic ones of the group of all euclidean motions in $n$-space $E^{n}$ for $n \geqq 3$. $^{1,2)}$ In this paper, we shall make use of the results of the preceding paper [2], but notations are independent of those in [2].

$\S 1$. Preliminaries and main theorems. Let $\mathbf{G}$ be the group of all euclidean motions in $n$-space $E^{n}$. Then $\mathbf{G}$ has a compact subgroup $\mathbf{K} \cong S O(n)$ and a normal subgroup $\mathbf{V}$ isomorphic to the vector group $R^{n}$, and

$$
\left\{\begin{array}{l}
\mathbf{G}=\mathbf{V} \cdot \mathbf{K}, \quad \mathbf{V} \cap \mathbf{K}=\{e\} \quad(e=\text { the identity of } \mathbf{G}) \\
\mathbf{G} / \mathbf{V} \cong \mathbf{K} .
\end{array}\right.
$$

Let $X$ be the character group of $\mathbf{v}$, and $\chi_{0}$ be the identity of $X$; then $X \cong R^{n}$. Hereafter $g, g^{\prime}, \ldots$ denote elements of $\mathbf{G}$, especially $a, b, c, \ldots$ of $\mathbf{K}, x$, $y, \ldots$ of $\mathrm{V}$; and $\chi, \chi^{\prime}, \ldots$ - elements of $X . \quad(\%, x)$ denotes the value of character $\%$ at $x \in \mathrm{V}$. We denote by $M_{a}$ the orthogonal matrix which realize the element $a \in \mathbf{K}$ and by $M_{a}^{*}$ its conjugate matrix, and define that $M_{a} x$ means to operate $M_{a}$ to $x$ as a vector in $R^{n}$ while $a x$ and $x a$ mean the multiplications as elements of the group G. We shall denote briefly $\% a$ instead of $M_{a}^{*} \%$. Then, if

$$
x=\left(\begin{array}{c}
x_{1} \\
\vdots \\
x_{n}
\end{array}\right), \quad \chi=\left(\varkappa_{1}, \ldots, \chi_{n}\right) \text { and } M_{a}=\left(\begin{array}{c}
a_{11} \ldots \\
\ldots \ldots \\
\ldots \\
a_{n 1} \ldots \\
\ldots
\end{array}\right) \text {, }
$$

Received August 12, 1952.

1) The author wrote in [2] that it seemed to be difficult to solve such problem for $n \geqslant 3$. But he could solve this problem after he finished the proof-reading of the paper [2].

2) Prof. G. W. Mackey kindly informed to the author that the result of [2] was inculuded in the result of his paper [3] which the author had overlooked. Recently more general cases have been treated in [4] and [5]. However, the results of the papers [3], [4] and [5] seem to be not so explicit as the result of our present paper. 
we have

$$
\left(\%, M_{a} x\right)=(\% a, x)=\exp \left(\sqrt{-1} \sum_{i j} a_{i j} \chi_{i} x_{j}\right) .
$$

$\tilde{X}=X-\left\{\psi_{0}\right\}$ is the product space of the unit sphere $S=S^{n-1}$ in $R^{n}$ and $T=\{0<t<\infty\}$ as topological spaces; we denote $\% \in \widetilde{X}$ by $\%=\langle s, t\rangle(s \in S$, $t \in T)$. Then $\% a=\langle s a, t\rangle$ by the above definitions.

$S$ may be considered as the factor space $\mathbf{K} / \mathbf{K}^{\prime}$ of right $\mathbf{K}^{\prime}$-cosets where $\mathbf{K}^{\prime} \cong S O(n-1)$. Hereafter $a^{\prime}, b^{\prime}, c^{\prime}, \ldots$ denote elements of $\mathbf{K}^{\prime}$. We shall denote by $s_{b}$ the image of $b \in \mathbf{K}$ under the natural mapping of $\mathbf{K}$ cnto $S$. For every $s \in S$, we fix an inverse image $c_{s}$ of $s$ under the natural mapping, where we do not demand the B-measurability etc. of the mapping $s \rightarrow c_{s}$. Every $b \in \mathbf{K}$ is uniquely expressible in the form $b=b^{\prime} c_{s}, b^{\prime} \in \mathbf{K}^{\prime}$, as far as the system $\left\{c_{s}\right\}$ is fixed. We shall consider the Haar measures $d b$ on $\mathbf{K}$ and $d b^{\prime}$ on $\mathbf{K}^{\prime}$ and the measure $d s$ on $S$ invariant under $\mathbf{K}$ such that

$$
d s \cdot d b^{\prime}=d b^{3)}
$$

Let $\left\{\widetilde{U}^{\lambda}\left(a^{\prime}\right)=\left\|\tilde{u}_{p q}^{\lambda}\left(a^{\prime}\right)\right\|(p, q=1, \ldots, \widetilde{n}(\lambda)) ; \lambda=1,2, \ldots\right\}$ be a system of irreducible unitary representaticns of the compact group $\mathbf{K}^{\prime}$ constructed by selecting a unitary representation from each class of mutually equivalent irreducible representations of $\mathbf{K}^{\prime}$, and $\left\{U^{\alpha}(a)=\left\|u_{i j}^{\alpha}(a)\right\|(i, j=1, \ldots, n(\alpha))\right.$; $\alpha=1,2, \ldots\}$ be a system of irreducible unitary representations of the compact group $\mathbf{K}$ constructed by the same method as above. Then $L^{\alpha}\left(a^{\prime}\right), a^{\prime} \in \mathbf{K}^{\prime}$, may be considered as a unitary representation of $\mathbf{K}^{\prime}$ and hence, by the complete reducibility, we may assume that $U^{\alpha}\left(a^{\prime}\right)$ is of the form:

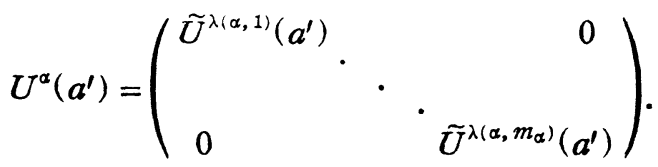

We fix such systems $\left\{U^{\alpha}(a)\right\}$ and $\left\{\tilde{U}^{\lambda}\left(a^{\prime}\right)\right\}$. We denote the number $\tilde{n}(\lambda(\alpha, 1))$ $+\ldots+\tilde{n}(\lambda(\alpha, m-1))$ by $N_{m}(\alpha)$ or simply by $N_{m}\left(m=1, \ldots, m_{\alpha}\right)$. Hereafter $i, j, k$ run over $\{1, \ldots, n(\alpha)\}$ while $p, q, r-\{1, \ldots, \tilde{n}(\lambda(\alpha, m))\}$ for $\alpha$ and $m$ being considered. Then, if $\mu=\lambda(\alpha, m)$, we have

$$
u_{N_{m}+p, j}^{\alpha}\left(b^{\prime} a\right)=\sum_{q} \tilde{u}_{p q}^{\mu}\left(b^{\prime}\right) u_{N_{m}+q, j}^{\alpha}(a)
$$

We put for any $\lambda$ and $p$

$$
\mathfrak{S}_{p}^{\lambda}=\left\{\begin{array}{l}
u_{N_{m}+p, j}^{\alpha}(b) / \begin{array}{l}
j=1, \ldots, n(\alpha), \text { and }\langle\alpha, m\rangle \text { runs over } \\
\text { all couples such that } \lambda(\alpha, m)=\lambda
\end{array}
\end{array}\right\}
$$

and

$$
\mathscr{S}_{p}^{\lambda}=\mathfrak{Q}\left[\Theta_{p}^{\lambda}\right]
$$

\footnotetext{
3) For the precise meaning of this equality, see [6], pp. 42-45.
} 
where $\Omega[\cong]$ denotes the closed linear subspace of $L^{2}(\mathbf{K})$ spanned by $\cong$. Then $\mathfrak{\subseteq}_{p}^{\lambda}$ is a complete orthogonal basis in $\aleph_{p}^{\lambda}$, and

$$
L^{2}(\mathbf{K})=\bigoplus_{\lambda=1}^{\infty} \tilde{n}_{p=1}^{\tilde{n}(\lambda)} \mathfrak{S}_{p}^{\lambda}
$$

Making use of these notions, we state here the main theorems.

Theorem 1.1. Fix an arbitrary element $t \in T$ and natural numbers $\lambda$ and $p(1 \leqq p \leqq \tilde{n}(\lambda))$, and define unitary operators $U_{t}(g), g \in \mathbf{G}$, in the Hilbert spa:e $\mathfrak{S}_{p}^{\lambda} b y$

$$
U_{t}(g) f(b)=U_{t}(x a) f(b)=\left(\left\langle s_{b}, t\right\rangle, x\right) f(b a) \quad\left(f \in \mathscr{S}_{p}^{\lambda} \subset L^{2}(\mathbf{K})\right)
$$

for $g=x a{ }^{4}$ Then $\left\{\mathscr{S}_{p}^{\lambda}, U_{t}(g)\right\}$ is an irreducible unitary representation of $\mathbf{G}$; and, for any sequence of complex numbers: $\left\{\hat{\varsigma}_{j}^{\alpha m} / j=1, \ldots, n(\alpha) ; \lambda(\alpha, m)=\lambda\right\}$ such that $\sum_{\lambda(a, m)=\lambda} \sum_{j}\left|\xi_{j}^{\alpha m}\right|^{2}=1$, the function

$$
\begin{aligned}
\Phi(g) \equiv \emptyset(x a) \\
=\int_{S}(\langle s, t\rangle, x)\left\{\sum_{\lambda(\alpha, m)=\lambda(\beta, l)=\lambda} \sum_{j k} \overline{\xi_{j}^{\alpha m}} \overline{\xi_{k}^{\beta l}} \times\right. \\
\left.\quad \times \sum_{r i} u_{v_{m}+r, i}^{\alpha}\left(c_{s}\right) u_{i j}^{\alpha}(a) \overline{u_{v_{l}+r}^{3}, k}\left(c_{s}\right)\right\} d s^{5)}
\end{aligned}
$$

is a normal elementary ${ }^{6)}$ p. $d_{0^{7}}$ function on $\mathbf{G}$ corresponding to the above irreducible unitary representation.

1.2. For any fixed $t$ and $\lambda$, the unitary representations $\left\{\hat{S}_{p}^{\lambda}, U_{t}(g)\right\}$ (defired in 1.1). $p=1, \ldots, \bar{n}(\lambda)$, are mutually unitary equivalent; while $\left\{\mathscr{S}_{p}^{\lambda}, U_{t}(g)\right\}$ and $\left\{\mathfrak{S}_{q}^{u}, U_{t}(g)\right\}$ are not mutually unitary equivalent for any $p$ and $q$ if $\lambda \neq \mu$.

1.3. If $t_{1} \neq t_{3}$, then $\left\{\mathscr{S}_{p}^{\lambda}, U_{t_{1}}(g)\right\}$ and $\left\{\mathcal{S}_{q}^{\mu}, U_{t_{2}}(g)\right\}$ are not mutually unitary equivalent for any $\lambda$, "l and $p, q$.

1.4. Put $\widetilde{\mathfrak{g}} \tilde{k} \equiv \mathrm{Q}\left[\left\{\boldsymbol{u}_{k j}^{\alpha}(b) / j=1, \ldots, n(\alpha)\right\}\right]$ for any fixed $\alpha$ and $k(1 \leqq k \leqq$ $n(\alpha)$ ), and define the unitaiy operator $U(g)$ in ${\widetilde{\mathfrak{F}_{k}}}_{k}^{\alpha}$ by

$$
U(g) f(b)=U(x a) f(b)=U(a) f(b)=f(b a) \quad\left(f \in \widetilde{\mathfrak{H}}_{k}^{\alpha} \subset L^{2}(\mathbf{K})\right)
$$

for $g=x a$. Then $\left\{\tilde{\mathscr{F}}_{k}^{x}, U(g)\right\}$ is an irreducible unitary representation of $\mathbf{G}$; and

$$
\Phi(g)=\emptyset(x a)=\sum_{i j} \xi_{i} \bar{\xi}_{j} u_{i j}^{\alpha}(a), \quad \sum\left|\hat{\xi}_{i}\right|^{2}=1,
$$

is a corresponding normal elementary p. d. function on $\mathbf{G}$.

4) Any element $g \in \mathbf{G}$ is uniquely expressible in this form by virture of (1.1).

5) The function in \{\} in the right-hand side is a B-measurable function of $s$ independent of the special choice of the system $\left\{c_{s}\right\}$; - see Lemma 1 ( $\left.\$ 2\right)$.

6) See [1], $\$ 15$.

7) p. d.=positive definite. 
1.5. $\left\{\tilde{\mathscr{S}}_{k}^{\alpha}, U(g)\right\}, k=1, \ldots, n(\alpha)$, are mutually unitary equivalent for any $\alpha$; while, if $\alpha \neq \beta,\left\{\widetilde{\mathscr{g}}_{k}^{\alpha}, U(g)\right\}$ and $\left\{\widetilde{\mathfrak{2}}_{j}^{\beta}, U(g)\right\}$ are not mutually unitary equivalent for any $k$ and $j$.

1.6. Every irreducible unitary representation of $\mathbf{G}$ is unitary equivalent to one of the above stated types. Consequently any normal elementary $p$. d. function on $\mathbf{G}$ is expressible in the form (1.7) or (1.9).

Theorem 2. Let $\sigma$ be the Haar measure on the compact group $\mathbf{K}$ and $\rho$ be a measure on $T$ such that $\rho(T)<\infty$, and define the unitary operator $U(g)$, $g \in \mathbf{G}$, in the Hilbert space $L^{2} \equiv L^{2}(\mathbf{K} \times T, \sigma \otimes \rho)^{8)}$ by

$$
U(g) f(b, t)=U(x a) f(b, t)=(\langle s b, t\rangle, x) f(b a, t) \quad\left(f \in L^{2}\right)
$$

for $g=x a$.

2.1. Let $\Delta_{\nu}^{\lambda}, \nu=1, \ldots, N(\lambda)(\leqq \infty) ; \lambda=1,2, \ldots$, be subsets of $T$ such that $\rho\left(\Delta_{\nu}^{\lambda}\right)>0$, and $\mathfrak{M}_{\nu}^{\lambda}$ be the totality of functions $\varphi(b, t)$ on $\mathbf{K} \times \Delta_{\nu}^{\lambda}$ of the form:

$$
\left.\varphi(b, t)=\sum_{\lambda(\alpha, m)=\lambda} \sum_{j} u_{N m+1, j}^{\alpha}(b) \varphi_{j}^{\alpha m}(t) \quad \text { (convergence in } L^{2}\right)
$$

where

$$
\sum_{\lambda,(\alpha, m)=\lambda} \sum_{\rho} \int_{\Delta_{\nu}^{\lambda}}\left|\varphi_{j}^{\alpha m}(t)\right|^{2} d \rho(t)<\infty .
$$

Then $\mathfrak{M}^{\lambda}$ is a closed linear subspace of $L^{2}$ invariant under $U(g), g \in \mathbf{G}$.

2.2. Let $\left\{f_{2, j}^{\alpha m}(t) / j=1, \ldots, n(\alpha) ; \lambda(\alpha, m)=\lambda ; \nu=1, \ldots, N(\lambda) ; \lambda=1\right.$, $2, \ldots\}$ be a sequence of functions satisfying:

$\left.1^{\circ}\right) \quad \sum_{\lambda} \sum_{\nu} \sum_{\lambda(\alpha, m)=\lambda} \sum_{j} \int_{\partial_{\nu}^{\lambda}}\left|f_{\nu j}^{\alpha m}(t)\right|^{2} d \rho(t)<\infty$,

$\left.2^{\circ}\right) \sum_{\lambda(\alpha, m)=\lambda} \sum_{\rho}\left|f_{\nu j}^{a m}(t)\right|^{2}>0$ for $\rho-a$. a. $t \in \Delta_{\nu}^{\lambda} \quad$ (a. a. = almost all $)$,

$\left.3^{\circ}\right)$ for any fixed $\lambda$, there is no function $\psi_{\nu}(t)$ for $\nu \neq \nu^{\prime}$ as follows: $f_{\nu j}^{\alpha m l}(t)=\psi_{w,}(t) f_{\nu^{\prime} j}^{\alpha m}(t)$ for all $j$ and all $\langle\alpha, m\rangle(\lambda(\alpha, m)=\lambda)$ for $\rho-a$. a. $t \in \Delta_{\imath}^{\lambda} \cap \Delta_{\imath}^{\lambda}$;

and put

$$
\left.f_{i}^{\lambda}(b, t)=\sum_{\lambda(\alpha, m)=\lambda} \sum_{j} u_{\Delta \dot{m}_{m}+1, j}^{\alpha}(b) f_{\nu j}^{\alpha m}(t) \quad \text { (convergence in } L^{2}\right) .
$$

Put $\mathfrak{N}_{\nu}^{\alpha}=\widetilde{\mathscr{S}}_{1}^{\alpha}$ (defined in Theorem 1.4) for $\nu=1, \ldots, N^{\prime}(\alpha)(\leqq \infty)$ and define unitary operators $U(g), g \in \mathbf{G}$, by $(1.8)$ and let $\left\{\xi_{i j}^{\alpha} / j=1, \ldots, n(\alpha) ; \nu=1, \ldots\right.$ $\left.N^{\prime}(\alpha), \alpha=1,2, \ldots\right\}$ be a sequence as follows:

$\left.4^{\circ}\right) \quad \sum_{\alpha} \sum_{\nu} \sum_{j}\left|\xi_{\nu j}^{\alpha}\right|^{2}<\infty$,

8) $\sigma \widehat{x} \rho$ denotes the product measure of $\sigma$ and $\rho$. 
$\left.5^{\circ}\right) \quad \sum_{j}\left|\xi_{\nu j}^{\alpha}\right|^{2}>0$ for any $\alpha$ and $\nu$,

$\left.6^{\circ}\right)$ for any fixed $\alpha$, there is no constant $\eta_{\nu \nu^{\prime}}$ for $\nu \neq \nu^{\prime}$ such that $\xi_{v j}^{\alpha}$ $=\eta_{\nu}, \xi_{\nu, j}^{\alpha}$ for any $j$;

and put

$$
h_{\nu}^{\alpha}(b)=\sum_{j} \xi_{\nu j}^{\alpha} u_{1 j}^{\alpha}(b)
$$

Let $\{\lambda\}^{\prime}$ and $\{\alpha\}^{\prime}$ be subsequences of the sequence $\{1,2, \ldots\}$ and define the unitary representation $\{\mathscr{g}, U(g)\}$ of $\mathbf{G}$ as the direct sum;

$$
\{\mathfrak{g}, U(g)\}=\left[\bigoplus_{(\lambda)^{\prime}} \oplus_{\nu}\left\langle\mathfrak{M}_{\nu}^{\lambda}, U(g)\right\}\right] \oplus\left[\bigoplus_{\{\alpha\}^{\prime}} \bigoplus_{\nu}\left\{\mathfrak{R}_{\nu}^{\alpha}, U(g)\right\}\right]
$$

and put

$$
f^{0}=\sum_{\langle\lambda\}^{\prime}} \sum_{\nu} f_{\nu}^{\lambda}+\sum_{\left\{x \gamma^{\prime}\right.} \sum_{v} h_{\nu}^{\alpha} .
$$

Then $\left\{\mathfrak{S}, U(g), f^{0}\right\}$ is a cyclic unitary representation of $\mathbf{G}$; the corresponding p. d. function $\Psi(\mathrm{g})$ is expressible as follows:

$$
\begin{aligned}
& \Psi(g) \equiv \Psi(x a) \\
& =\sum_{\left\{\lambda \gamma^{\prime}\right.} \sum_{\nu} \int_{\Delta_{\nu}^{\lambda}} d \rho(t) \int_{S} \int_{\lambda(\alpha, m)=\lambda(\beta, l)=\lambda} \sum_{j k} f_{\nu j}^{\alpha m}(t) f_{\nu k}^{\beta l}(t) \times \\
& \times(\langle s, t\rangle, x) \sum_{r i} u_{v_{m}+r, i}^{\alpha}\left(c_{s}\right) u_{i j}^{\alpha}(a) \overline{u_{N_{l}+r, k}^{\beta}\left(c_{s}\right)} d s \\
& +\sum_{i \alpha \gamma^{\prime}} \sum_{\nu} \sum_{i j} \xi_{\nu j}^{\alpha} \xi_{\nu i}^{\alpha} u_{i j}^{\alpha}(a) \text {. }
\end{aligned}
$$

2.3. If we replace $u_{v_{m}+1, j}^{\alpha}(b)$ in the definition of $\mathfrak{M i}_{i}$ in 2.1 by $\boldsymbol{u}_{i i_{m}+p, j}^{\alpha}(b)$ and ${\widetilde{\oiint_{1}}}_{1}^{\alpha}$ in 2.2 by $\widetilde{\mathscr{S}}_{k}^{\alpha}$ where $p$ may depend on $\nu$ and $\lambda=\lambda(\alpha, m)$, and $k$-on $\alpha$ and $\nu$, then we obtain a cyclic unitary representation of $\mathbf{G}$ which is uni_ary equivalent to the original one.

2.4. Every cyclic unitary representation of $\mathbf{G}$ is unitary equivalent to that of above staied type, and any $p . d$. function on $\mathbf{G}$ is expressible in the form (1. 12).

THeORem 3. (Generalization of Bochner's theorem) Any p.d. funtion $\psi(\mathrm{g})$ on $\mathrm{G}$ is expressible by means of normal elementary $p$. $d$. functions in the following form:

$$
\Psi(g)=\sum_{\lambda=1}^{\infty} \sum_{\nu=1}^{\infty} \xi_{\nu}^{\lambda} \int_{\Delta_{\nu}^{\lambda}} \Phi_{\nu}^{\lambda}(g ; t) d \rho(t)+\sum_{\alpha=1}^{\infty} \sum_{\nu=1}^{\infty} \eta_{\nu}^{\alpha} \Phi_{\nu}^{\alpha}(g)
$$

where $\Phi_{\nu}^{\lambda}(g, t)$ and $\Phi_{\nu}^{x}(g)$ are normal elementary $p$. d. funstions (cf. (1.7), (1.9) and (1.12)), $\Delta_{\nu}^{\lambda} \subset T$ and $\xi_{\nu}^{\lambda}, \eta_{\nu}^{\alpha} \geq 0, \sum_{\lambda \nu} \xi_{i}^{\lambda} \rho\left(\Delta_{\nu}^{\lambda}\right)<\infty, \sum_{\alpha \nu} \eta_{\nu}^{\alpha}<\infty$.

We shall prove these theorems in $\S 4$ by making use of results of $\S \S 2$ and 3 . 
Remark. The argument in this paper may be applied to any Lie group $\mathbf{G}$ of the following type: $\mathbf{G}$ has a closed normal subgroup $\mathbf{V}$ isomorphic to a vector group and the factor group $\mathbf{G} / \mathbf{V}$ is compact.

§2. Unitary representations of $\mathbf{G}$ in $L^{2}(\mathbf{K})$. We fix an element $t_{i} \in T$ and denote $\left(\left\langle s, t_{0}\right\rangle, x\right)$ by $(s, x)$ briefly, and define unitary operators $U(g), g$ $\in \mathbf{G}$, in the Hilbert space $L^{2}(\mathbf{K})$ as follows:

$$
U(g) f(b)=U(x a) f(b)=(s b, x) f(b a) \quad\left(f \in L^{2}(\mathbf{K})\right) \text { for } g=x a .
$$

We shall use notations defined in $\S 1$, but, in this paragraph, (.,.) and $\|$. denote respectively the inner product and the norm in $L^{2}(\mathbf{K})$.

The following lemma may be verified by making use of (1.4) and the orthogonality-relation of the system $\left\{\tilde{u}_{p q}^{\lambda}\left(b^{\prime}\right)\right\}$ in $L^{2}\left(\mathbf{K}^{\prime}\right)$.

LEMMA 1. For any $a \in \mathbf{K}$ and any $s \in S$, it holds that

$$
\begin{aligned}
& \int_{\mathbf{K}^{\prime}} u_{N_{m}+p, j}^{\alpha}\left(b^{\prime} c_{s} a\right) \overline{u_{N_{l}+q, k}^{3}\left(b^{\prime} c_{s}\right)} d b^{\prime} \\
& = \begin{cases}\sum_{r_{i}} u_{N_{m}+r, j}^{\alpha}\left(c_{s}\right) u_{i, j}^{\alpha}(a) u_{k, N_{m}+r}^{\mathrm{R}}\left(c_{s}^{-1}\right) / \tilde{n}(\lambda(\alpha, m)) \\
0 & \text { if } \lambda(\alpha, m)=\lambda(\beta, l) \text { and } p=q,\end{cases}
\end{aligned}
$$

and consequently, for any a, the function of the form in the right-hand side of above equality is a B-measurable function of $s$ independent of the special choice of the system $\left\{c_{s}\right\}$ (see $\left.\S 1\right)$.

Next, if we put $\overline{\mathscr{S}}_{p}^{\lambda}=\Omega\left[\left\{U(g) f / f \in \mathscr{S}_{b}^{\lambda}, g \in \mathbf{G}\right\}\right]$, then we have

LEMMA 2. If $\lambda \neq \mu$ or $p \neq q$, then $\overline{\mathfrak{S}}_{p}^{\lambda}$ and $\bar{\aleph}_{q}^{\mu}$ are mutually orthogonal in $L^{2}(\mathbf{K})$.

Proof. It is sufficient to prove that $(U(g) \varphi, \psi)=0$ for any $\varphi \in \mathfrak{S}_{p}^{\lambda}, \psi \in \mathfrak{S}_{q}^{\mu}$ and any $g \in \mathbf{G}$. $\varphi, \psi$ and $g$ are expressible in the form:

$$
\varphi=\sum_{\lambda(\alpha, m)=\lambda} \sum_{j} \xi_{j}^{\alpha m} u_{v_{m}+p, j}^{\alpha}, \quad \psi=\sum_{\lambda(\beta, l)=\mu^{\prime}} \sum_{k} \eta_{k}^{\beta l} u_{v_{l}+q, k}^{\beta}, \quad g=x a .
$$

Hence we have

$$
\begin{aligned}
(U(g) \varphi, \psi) & =\int_{\mathbf{K}}(s, x) \varphi(b a) \overline{\psi(b)} d b \\
& =\int_{S}(s, x) d s \int_{\mathbf{K}^{\prime}} \varphi\left(b^{\prime} c_{s} a\right) \overline{\psi\left(b^{\prime} c_{s}\right)} d b^{\prime}=0
\end{aligned}
$$

by (1.2) and Lemma 1, q.e.d.

COROLLARY. $\quad \overline{\mathscr{S}}_{p}^{\lambda}=\mathscr{S}_{p}^{\lambda}$; consequently $\mathscr{S}_{\partial p}^{\lambda}$ is a subspace of $L^{2}(\mathbf{K})$ invariant under $U(g), g \in \mathbf{G}$. 
This fact is proved from (1.5) and Lemma 2.

LEMMA 3. For any given $\lambda$ and $p$, we fix a couple $\langle\alpha, m\rangle$ such that $\lambda(\alpha$, $m)=\lambda$ and put $k=N_{m}(\alpha)+p$. If $\varphi \in \mathfrak{S}_{p}^{\lambda}$ and if the p. d. function $(U(g) \varphi, \varphi)^{9)}$ on $\mathbf{G}$ is a minorant ${ }^{10)}$ of the p.d.function $\left(U(g) u_{k k}^{r}, 2 i_{k k}^{r}\right)$, then $\varphi=\xi u_{k k}^{\tilde{r}}$, $\xi b e-$ ing a complex number.

Proof. By the assumption and by Corollary. of Lemma 2, there exists an element $\psi \in \mathfrak{S}_{\alpha}^{\lambda}$ such that

$$
(U(g) \varphi, \varphi)+(U(g) \psi, \psi)=\left(U(g) u_{k k}^{\alpha}, u_{k k}^{\alpha}\right),
$$

especially, putting $g=a \in \mathbf{K}$, we have

$$
\int_{\mathbf{K}} \varphi(b a) \overline{\varphi(b)} d b+\int_{\mathbf{K}} \psi(b a) \overline{\psi(b)} d b=u_{k k}^{\tilde{q}}(a) / n(\alpha) .
$$

Each term of the left-hand side is p. d. function of $a(\in \mathbf{K})$, while $u_{k k}^{\alpha}(a)$ is an elementary $p$. $d$. function on $\mathbf{K}$. Hence we have ${ }^{11)}$

$$
\left.\begin{array}{l}
\int_{\mathbf{K}} \varphi(b a) \overline{\varphi(b)} d b=\eta u_{k k}^{\alpha}(a) / n(\alpha) \\
\int_{\mathbf{K}} \psi(b a) \overline{\psi(b)} d b=(1-\eta) u_{k k}^{\alpha}(a) / n(\alpha)
\end{array}\right\} \quad 0<\eta<1
$$

On the other hand, $\varphi$ is expressible in the form:

$$
\varphi=\sum_{\lambda,\left(\beta, l_{i}=\lambda\right.} \sum_{j} \xi_{j}^{3 l} u_{x_{l}+p, j}^{3} .
$$

Hence it follows from the orthogonality-relation of $\left\{u_{i j}^{\mathrm{R}}(b)\right\}$ that

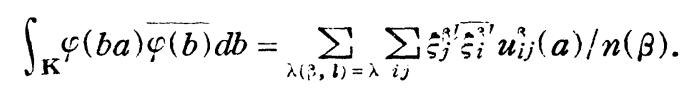

From this equality and (2.2), we get

$$
\sum_{l}^{\lambda(3, l)=\lambda} \mid \xi_{j}^{3 l ! 2}=\eta \delta_{\alpha \beta} \delta_{k j} \quad(\delta: \text { Kronecker's delta })
$$

where $\sum_{l}^{\lambda(3, l)=\lambda}$ means the summation for all $l$ such that $\lambda(\beta, l)=\lambda$ for fixed $\beta$. Hence may be expressible as follows:

$$
\varphi(b)=\sum_{l}^{\lambda(\alpha, l)=\lambda} \xi_{l} u_{\Delta_{l}+p, k}^{\alpha}(b), \quad \sum_{l}^{\lambda(\alpha, l=\lambda} \mid \xi l^{2}=\eta
$$

Similarly we get

9) See [1], \&7.

10) See [1], $\S 11$; - of couse, we do not mean the trivial one: the function identically equal to zero.

11) See Theorem 7 in [1]. 


$$
\psi(b)=\sum_{l}^{\lambda(\alpha, l)=\lambda} \eta_{l} u_{N_{l}+p, k}^{\alpha}(b) .\left.\quad \sum_{l}^{\lambda(a, l)=\lambda}|\eta|\right|^{2}=1-\eta .
$$

Consequently

$$
\sum_{l}^{\lambda(\alpha, l)=\lambda}\left\{|\xi \xi|^{2}+\left.|\eta|\right|^{2}\right\}=1
$$

If we put $g=x \in \mathbf{V}$ in (2.1), we have (by (1.2))

$$
\begin{gathered}
\int_{s}(s, x) d s \int_{\mathbf{K}^{\prime}}\left|\psi\left(b^{\prime} c_{s}\right)\right|^{2} d b^{\prime}+\int_{s}(s, x) d s \int_{\mathbf{K}^{\prime}}\left|\psi\left(b^{\prime} c_{s}\right)\right|^{2} d b^{\prime} \\
=\int_{s}(s, x) d s \int_{\mathbf{K}^{\prime}}\left|u_{k k}^{\alpha}\left(b^{\prime} c_{s}\right)\right|^{2} d b^{\prime} .
\end{gathered}
$$

Since $\psi(b)$ and $\psi(b)$ are continuous by (2.3) and $\left(2.3^{\prime}\right)$, and since $x(\in \mathbf{V})$ is arbitrary in the above equality, we obtain for any $s \in S$

$$
\int_{\mathbf{K}^{\prime}}\left|\varphi\left(b^{\prime} c_{s}\right)\right|^{2} d b^{\prime}+\int_{\mathbf{K}^{\prime}}\left|\psi\left(b^{\prime} c_{s}\right)\right|^{2} d b^{\prime}=\int_{\mathbf{K}^{\prime}}\left|\boldsymbol{u}_{k k}^{\alpha}\left(b^{\prime} c\right)\right|^{2} d b^{\prime}
$$

Putting $s=s_{e}$ (whence we may put $c_{s}=e$ ) in this equality, we have

$$
\begin{aligned}
\int_{\mathbf{K}^{\prime}}\left|\varphi\left(b^{\prime}\right)\right|^{2} d b^{\prime}+\int_{\mathbf{K}^{\prime}}\left|\psi\left(b^{\prime}\right)\right|^{2} d b^{\prime} & =\int_{\mathbf{K}^{\prime}}\left|u_{k k}^{\alpha}\left(b^{\prime}\right)\right|^{2} d b^{\prime} \\
& =\tilde{u}_{p p}^{\lambda}(e) / \widetilde{n}(\lambda) \neq 0 .
\end{aligned}
$$

By (1.3) and by the assumption: $k=N_{m}(\alpha)+p$,

$$
u_{N_{l}+p, k}^{\alpha}\left(b^{\prime}\right) \equiv 0 \text { on } \quad \mathbf{K}^{\prime} \text { for } l \neq m .
$$

Hence, from $(2.3),\left(2,3^{\prime}\right)$ and $(2.5)$, we get

$$
\left|\hat{\xi}_{m}\right|^{2}+\left|\eta_{m}\right|^{2}=1 \text {. }
$$

From this and (2.4), we obtain $\xi_{l}=\eta_{l}=0$ for $l \neq m$, and hence $\varphi=\xi_{m} u_{N_{m}+\not, k}^{\alpha}$ by (2.3), q.e.d.

Lemma 4. Let $\alpha, m$ and $k$ be as in Lemma 3 for any given $\lambda$ and $p$. Then $\left\{\xi_{p}^{\lambda}, U(g), u_{k k}^{\alpha}\right\}$ is a cyclic unitary representation of $\mathbf{G}$.

Proof. For any $\beta, l$ and any $i, j(1 \leqq i, j \leqq n(\beta))$ it holds that

$$
u_{N l+p, i}^{3} \in \mathbb{Q}\left[\left\{U(a) u_{. v_{l}+p, j}^{3} / a \in \mathbf{K}(\subset \mathbf{G})\right\}\right]
$$

by the irreducibility of $U^{3}(a)$ as a representation of $\mathbf{K}$. By virtue of this fact and Corollary of Lemma 2 , it suffices to prove that $\lambda(\beta, l)=\lambda$ implies

$$
u_{N_{l}+p, 1}^{\mathrm{R}} \in \mathbb{Q}\left[\left\{U^{\top}(g) u_{k j}^{\alpha} / j=1, \ldots, n(\alpha) ; g \in \mathbf{G}\right\}\right] .
$$

Now, if $\lambda(\beta, l)=\lambda=\lambda(\alpha, m)$, then, by Lemma 1 , the functions $f j(s)(j=1$, $\ldots, n(\alpha))$ defined by 


$$
\begin{aligned}
\varphi_{j}(s) & \equiv \tilde{n}(\lambda) \int_{\mathbf{K}^{\prime}} u_{N^{\prime} l+p, 1}^{\beta}\left(b^{\prime} c_{s}\right) \cdot \overline{u_{N_{m}+p, j}^{\mathrm{\alpha}}\left(b^{\prime} c_{s}\right)} d b^{\prime} \\
& =\sum_{q} u_{N_{l}+q, 1}^{\mathrm{\beta}}\left(c_{s}\right) u_{j, s_{m}+q}^{\alpha}\left(c_{s}^{-1}\right)
\end{aligned}
$$

are bounded B-measurable functions on $S$ and it holds for any $r(1 \leqq r \leqq \widetilde{n}(\lambda))$ and any $s \in S$ that

$$
\begin{aligned}
\sum_{J} u_{v_{m}+r, j}^{\alpha}\left(c_{s}\right) \varphi_{j}(s) & =\sum_{\alpha} \sum_{0} u_{N_{m}+r, j}^{\alpha}\left(c_{s}\right) u_{j, v_{m}+q}^{\alpha}\left(c_{s}^{-1}\right) u_{v_{l}+q, 1}^{\beta}\left(c_{s}\right) \\
& =\sum_{\boldsymbol{q}} u_{\Delta_{m}+r, v_{m}+q}^{\alpha}(e) u_{v_{l}+q, 1}^{\beta}\left(c_{s}\right)=u_{v_{l}+r, 1}^{\mathrm{\beta}}\left(c_{s}\right) .
\end{aligned}
$$

Hence, by means of the relation: $u_{N_{l}+p, N_{l}+q}^{\beta}\left(b^{\prime}\right)=\tilde{u}_{p q}^{\lambda}\left(b^{\prime}\right)=u_{N_{m}+p, N_{m}+q}^{\alpha}\left(b^{\prime}\right)$, we get ( for $b=b^{\prime} c_{s}$ )

$$
\begin{aligned}
u_{N_{l}+p, 1}^{\beta}(b) & =\sum_{r} u_{N_{l}+p, N_{l}+r}^{\beta}\left(b^{\prime}\right) u_{N_{l}+r, 1}^{\beta}\left(c_{s}\right) \\
& =\sum_{r i} u_{N_{m}+p, N_{m}+r}^{\alpha}\left(b^{\prime}\right) u_{N_{m}+r, j}^{\alpha}\left(c_{s}\right) \varphi_{j}(s)=\sum_{j} u_{N_{m}+p, j}^{\alpha}(b) \varphi_{j}(s) .
\end{aligned}
$$

On the other hand, there exist complex numbers $\xi_{j,}$ and elements $x_{j \nu}$ of $\mathbf{V}(\nu$ $=1, \ldots, N(j))$ for any $\varepsilon>0$ and every $j$ such that

$$
\int_{s}\left|\varphi_{j}(s)-\sum_{\nu} \xi_{j v} \cdot\left(s, x_{j,}\right)\right|^{2} d s<\varepsilon^{2} / n(\alpha)^{2}
$$

since $\varphi_{j}(s), j=1, \ldots, n(\alpha)$, are bounded and B-measurable on $S$. Therefore, by simple calculation, we get

$$
\left\|u_{N l+p, 1}^{\beta}-\sum_{j \downarrow} \xi_{j,} U\left(x_{j v}\right) \cdot u_{v_{m}+p, j}^{\alpha}\right\|<\varepsilon .
$$

This result shows (2.6), q.e.d.

PROPOSITION 1. $\left\{\boldsymbol{\xi}_{p}^{\lambda}, U(g)\right\}$ is an irreducible unitary representation of $\mathbf{G}$ for any $\lambda$ and $p(1 \leqq p \leqq \tilde{n}(\lambda))$.

This proposition is clear by Corollary of Lemma 2, Lemmas 3 and 4, and Theorem 7 in [1].

Corollary. i) If a unitary operator $U$ in $\mathfrak{g}_{p}^{\lambda}$ is permutable with any $U(g)$, $g \in \mathrm{G}$, then $U=\xi I,|\xi|=1$; consequently ii ) If $\varphi, \psi \in \xi_{p}^{\lambda}$ and $(U(g) \varphi, \varphi)=(U(g) \psi$, $\psi$ ) for any $g \in \mathbf{G}$, then $\psi=\xi \varphi,|\xi|=1$.

These are immediate results of Proposition 1.

Proposition 2. For any fixed $\lambda$, the unitary representations $\left\{\xi_{\alpha} \lambda, U(g)\right\}, p$ $=1, \ldots, \tilde{n}(\lambda)$, are mutuallv unitary equivalent.

Proof. We fix a couple $\langle\alpha, m\rangle$ such that $\lambda(\alpha, m)=\lambda$. Then $\left\{\mathscr{F}_{p}^{\lambda}, U(g)\right.$, $\left.u_{N_{m}+p, 1}^{\alpha}\right\}, p=1, \ldots, \widetilde{n}(\lambda)$, are cyclic unitary representations of $\mathbf{G}$ (by Lemma 
4). Hence it is sufficient to prove that p. d. functions $\left(U(g) u_{v_{m}+p, 1}^{\alpha}, u_{v_{1 m}+p, 1}^{\alpha}\right), p$ $=1, \ldots, \tilde{n}(\lambda)$, are mutually iden ical. For any $g=x a \in \mathbf{G}$, we have by (1.2) and Lemma 1

$$
\begin{aligned}
\left(U(g) u_{N_{m}+p, 1}^{\alpha}, u_{N_{m+}+p, 1}^{\alpha}\right) & =\int_{s}(s, x) d s \int_{\mathbf{K}^{\prime}} u_{N_{m}+p, 1}^{\alpha}\left(b^{\prime} c_{s} a\right) \overline{u_{N_{m}+p, 1}^{\alpha}\left(b^{\prime} c_{s}\right)} d b^{\prime} \\
& =\int_{s}(s, x)\left\{\sum_{q i} u_{N_{m}+q, i}^{\alpha}\left(c_{s}\right) u_{i 1}^{\alpha}(a) u_{1, s_{m}+q}^{\alpha}\left(c_{s}^{-1}\right) / \tilde{n}(\lambda)\right\} d s
\end{aligned}
$$

this is independent of $p$, q.e.d.

Proposition 3. If $\lambda \neq \mu$, then the unitary representations $\left\{\mathfrak{S}_{p}^{\lambda}, U(g)\right\}$ and $\left\{S_{q}^{\mu}, U(g)\right\}$ are not mutually unitary equivalent for any $p$ and $q$.

Proof. By Proposition 2, it suffices to prove this for $p=q=1$. We denote the operator $U(g)$ considered in $\mathscr{S}_{1}^{\lambda}$ and $\mathscr{S}_{1}^{\mu}$ by $U_{1}(g)$ and $U_{2}(g)$ respectively. If $\left\{\mathscr{S}_{1}^{\lambda}, U_{1}(g)\right\}$ is unitary equivalent to $\left\{\mathfrak{g}_{1}^{\mu}, U_{2}(g)\right\}$, then there exists a unitary transformation $U$ of $\xi_{1}^{\lambda}$ onto $\$_{1}^{\mu}$ such that $U_{2}(g)=U \cdot U_{1}(g) \cdot U^{-1}$. We fix a couple $\langle\alpha, m\rangle$ such that $\lambda(\alpha, m)=\lambda$, and put $k=N_{m}(\alpha)+1$. Then $u_{k k}^{\alpha} \in \mathscr{F}_{1}^{\lambda}$ and $f=U \cdot u_{k k}^{\alpha} \in \mathfrak{S}_{1}^{\mu}$. The element $f$ is expressible in the form $: f=\sum_{\lambda(\beta, l)=\mu} \sum_{j} \xi_{j}^{\beta l} u_{v_{l}+1, j}^{\beta}$, and hence for any $a^{\prime} \in \mathbf{K}^{\prime}$

$$
\begin{aligned}
& \left(U_{2}\left(a^{\prime}\right) f, f\right)=\sum_{\lambda\left(\beta, l, l=\mu_{i}\right.} \sum_{\iota j} \xi_{j}^{3 l} \xi_{i}^{\beta l} u_{i j}^{\beta}\left(a^{\prime}\right) / n(\beta) \\
& =\sum_{p q} \bar{u}_{p q}^{\mu}\left(a^{\prime}\right) \sum_{\lambda(\beta, l)=\mu} \xi_{N_{l}+q}^{\beta !} \overline{\xi_{N N_{l}+p}^{\beta !}} / n(\beta) \quad \text { (by (1.3)). }
\end{aligned}
$$

On the other hand

$$
\begin{aligned}
\left(U_{2}\left(a^{\prime}\right) f, f\right) & =\left(U \cdot U_{1}\left(a^{\prime}\right) \cdot U^{-1} f, f\right) \\
& =\left(U_{1}\left(a^{\prime}\right) u_{k k}^{\alpha}, u_{k k}^{\alpha}\right)=\tilde{u}_{11}^{\lambda}\left(a^{\prime}\right) / n(\alpha) .
\end{aligned}
$$

This is a contradiction, because $\lambda \neq \mu$ implies that $\tilde{u}_{p q}^{\mu}\left(a^{\prime}\right)$ and $\tilde{u}_{11}^{\lambda}\left(a^{\prime}\right)$ are mutually orthogonal in $L^{2}\left(\mathbf{K}^{\prime}\right)$ for any $p$ and $q$, q.e.d.

§ 3. Unitary representations of $\mathbf{G}$ in $L^{2}(\mathbf{K} \times T, \sigma \otimes \rho)$. Let $\Delta$ be a subset of $T$ and $\mathfrak{M}_{i p}^{\lambda}(\Delta)$ be the totality of functions $\varphi(b, t) \in L^{2} \equiv L^{2}(\mathbf{K} \times \Delta, \sigma \otimes \rho)$ of the form

$$
\varphi(b, t)=\sum_{\lambda(\alpha, m)=\lambda} \sum_{\rho} u_{\Delta m+p, j}^{\alpha}(b) \varphi_{p j}^{\alpha m}(t), \quad \sum \sum \int_{\Delta}\left|\varphi_{p j}^{\alpha m}(t)\right|^{2} d \rho(t)<\infty .
$$

We may prove easily the following

Lemia 5. Any function $\varphi(b, t) \in L^{2}(\mathbf{K} \times T, \sigma \otimes \rho)$ is uniquely expressible in the form:

$$
\left.\varphi(b, t)=\sum_{\mu} \sum_{p} \sum_{\lambda\left(\alpha, w_{i}\right)=\mu} \sum_{j} u_{\lambda_{m}+p, j}^{\alpha}(b) \varphi_{p j}^{\alpha m}(t) \quad \text { (convergence in } L^{2}\right)
$$


where

$$
\varphi_{p j}^{\alpha m}(t)=\int_{\mathbf{K}} \varphi(b, t) \overline{u_{v_{m}+p, j}^{\alpha}(b)} d b
$$

and consequently

$$
\sum_{\mu} \sum_{p} \sum_{\lambda(\alpha, m)=\mu} \sum_{j} \int_{T}\left|\varphi_{p j}^{\alpha m}(t)\right|^{2} d \rho(t)=\int_{\mathbf{K} \times T}|\varphi(b, t)|^{2} d b d \rho(t) .
$$

Profosition 4. $\mathbb{M}_{p}^{2}(\Delta)$ is a closed linear subspace of $L^{2}(\mathbf{K} \times T, \sigma \otimes \rho)$ invariant under $U(g), g \in \mathrm{G}$, defined in Theorem 2.1.

It is clear from the definition of $U(g)$ and by Lemma 2 that $\mathfrak{M}_{p}^{\lambda}(\Delta)$ is a linear subspace of $L^{2}(\mathbf{K} \times T, \sigma \notin \rho)$ invariant under $U(g), g \in \mathbf{G}$. The closedness of $\mathfrak{M}_{p}^{\lambda}(\Delta)$ may be proved by virtue of Lemma 4 .

Thus $\left\{\mathfrak{M}_{p}^{\prime}(d), \mathcal{L}(g)\right\}, p=1, \ldots, \tilde{n}(\lambda) ; \lambda=1,2, \ldots$, may be considered as unitary representations of $\mathbf{G}$.

Lemma 6. If $f_{1} \in \mathfrak{M}_{p}^{\lambda}\left(\Delta_{1}\right), f_{:} \in \mathfrak{M}_{p}^{\mu}\left(\Delta_{2}\right)$ and if p. d. functions $\left(U(g) f_{1}, f_{1}\right)$ and $\left(U(g) f_{2}, f_{2}\right)$ have a common minorant, ${ }^{12 ;}$ then there exist a Borel set $\Delta_{0} \subset \Delta_{1}$ $\cap \Delta_{2}$ such that $\rho\left(\Delta_{0}\right)>0$ and a B-measurable finction $\omega(t)$ defined on $\Delta_{0}$ such that $0<|\omega(t)|<\infty$ and $f_{1}(b, t)=\omega(t) f_{2}(b, t)$ for $\sigma-a$. a. $b \in \mathbf{K}$ for $\rho-a . a . t$ $\in J_{0} ;$ consequently $\lambda=\mu$.

Proof. Let $\Psi(g)$ be a common minorant of $\left(U(g) f_{1}, f_{1}\right)$ and $\left(U(g) f_{2}, f_{2}\right.$. Then, by Theorem 5 in $[1], \Psi(g)$ is expressible as follows:

$$
\Psi(g)=\left(U(g) \psi_{1}, \psi_{1}\right)=\left(U(g) \psi_{2}, \psi_{2}\right), \quad \psi_{1} \in \mathfrak{M}_{p}^{\lambda}\left(\Delta_{1}\right), \quad \phi_{2} \in \mathfrak{M}_{p}^{\mu}\left(\Delta_{2}\right) ;
$$

furthermore there exist $\varphi_{1} \in \mathfrak{M}_{p}^{\lambda}\left(\Delta_{1}\right)$ and $\varphi_{2} \in \mathfrak{M}_{p}^{\mu}\left(\Delta_{2}\right)$ such that

$$
\begin{aligned}
& \int_{\mathbf{K} \times T}\left(\left\langle s_{b}, t\right\rangle, y\right)\left(\left\langle s_{b}, t\right\rangle, x\right) f_{i}(b a, t) \overline{f_{y}(b, t)} d b d \rho(t) \\
& =\int_{\mathbf{K} \times T}\left(\left\langle s_{b}, t\right\rangle, y\right)\left\{\left(\left\langle s_{b}, t\right\rangle, x\right) \psi_{\nu}(b a, t) \overline{\psi_{\nu}(b, t)}+\right. \\
& \left.+\left(\left\langle s_{b}, t\right\rangle, x\right) \varphi_{,}(b a, t) \varphi_{,}(b, t)\right\} d b d \rho(t), \quad \nu=1,2,
\end{aligned}
$$

for any $y, x \in \mathbf{V}$ and $a \in \mathbf{K}$ (we put $f(b, t) \equiv 0$ on $\mathbf{K} \times\left(T-\Delta_{.}\right.$) for any function $\left.\in m_{b}^{\lambda}\left(\Delta_{\nu}\right)\right)$. For any Borel set $\Delta \subset T$, the characteristic function of the set $\mathbf{K} \times \Delta$ may be approximated in $L^{2}(\mathbf{K} \times T, \sigma \otimes \rho)$ by means of linear combinations of "characters" $\left(\left\langle s_{b}, t\right\rangle, y\right)$. Hence (3.5) implies that

$$
\int_{\mathbf{K}}(\langle s b, t\rangle, x) f_{\nu}(b a, t) f_{,}(b, t) d b
$$

$$
=\int_{\mathbf{K}}(\langle s b, t\rangle, x) \dot{\psi} \cdot(b a, t) \bar{\psi} \cdot(b, t) d b+
$$

12) See the foot-note 10 ). 


$$
+\int_{\mathbf{K}}(\langle s b, t\rangle, x) \varphi_{\nu}(b a, t) \varphi_{,}(b, t) d b, \quad \nu=1,2,
$$

for any $x \in \mathbf{V}_{0}$ and $a \in \mathbf{K}_{0}$ for $\rho-$ a. a. $t \in T$ where $\mathbf{V}_{0}$ and $\mathbf{K}_{0}$ are dense subsets of $\mathbf{V}$ and $\mathbf{K}$ respectively such that $\overline{\overline{\mathbf{V}}}_{0}=\overline{\overline{\mathbf{K}}}_{0}=\S_{0}$; and hence, by Lebesgue's convergence theorem, (3.6) is true for any $x \in \mathbf{V}$ and $a \in \mathbf{K}$ for $\rho-$ a. a. $t \in T$. Similar argument shows that (3.4) implies

$$
\begin{aligned}
\int_{\mathbf{K}}\left(\left\langle s_{b}, t\right\rangle, x\right) \psi_{1}(b a, t) \overline{\psi_{1}(b, t) d b} \\
\quad=\int_{\mathbf{K}}\left(\left\langle s_{b}, t\right\rangle . x\right) \psi_{2}(b a, t) \psi_{2}(b, t) d b
\end{aligned}
$$

for $\rho$-a. a. $t \in T$. Each term in (3.6) and (3.7) expresses a p. d. function of $g=x a$; especially the left-hand side of (3.6) expresses an elementary p. d. function corresponding to the irreducible unitary representation $\left\{\xi_{2}^{\lambda}, U_{t}(g)\right\}$ or $\left\{\aleph_{p}^{\mu}, U_{t}(g)\right\}$ stated in $\$ 2$ if $\nu=1$ or $\nu=2$ respectively. Hence, by Theorem 7 in $[1]$, there exists a function $\omega_{0}(t) \geqslant 0$ such that

$$
\begin{aligned}
\int_{\mathbf{K}}\left(\left\langle s_{b}, t\right\rangle, x\right) f_{1}(b a, t) \overline{f_{1}(b, t)} d b \\
=\omega_{0}(t) \int_{\mathbf{K}}\left(\left\langle s_{b}, t\right\rangle, x\right) f_{2}(b a, t) f_{2}(b, t) d b
\end{aligned}
$$

for any $x \in V$ and $a \in K$ for a. a. $t \in T$, and hence, by Proposition 3 and Corollary of Proposition 1, we obtain that $\lambda=\mu$ and that

$$
f_{1}(b, t)=\omega(t) f_{2}(b, t) \text { for } \sigma-\text { a. a. } b
$$

for $\rho$-a. a. $t$ for a certain $\omega(t)\left(|\omega(t)|^{2}=\omega_{0}(t)\right)$, which is B-measurable in $t$ by Fubini's theorem. If we put

$$
\Delta_{0}=\left\{t / \int_{\mathbf{K}}\left|\psi_{1}(b, t)\right|^{2} d b=\int_{\mathbf{K}}\left|\psi_{2}(b, t)\right|^{2} d b \neq 0\right\} \quad(\text { see }(3.7)),
$$

then we may easily show that the set $\Delta_{0}$ and the function $\omega(t)$, considered on $\Delta_{0}$, have the properties stated in Lemma 6, q.e.d.

Proposition 5. The unitary representations $\left\{\mathfrak{M}_{p}^{\lambda}(\Delta), U(g)\right\}$ and $\left\{\mathfrak{M}_{q}^{\lambda}(\Delta)\right.$, $U(g)\}$ are mutually unitary equivalent for any $p$ and $q(1 \leqq p, q \leqq \tilde{n}(\lambda))$.

This fact is easily verified from the definition of $\mathfrak{M}_{p}^{\lambda}(\Delta)$ and by Proposition 2.

Proposition 6. If $\lambda \neq \mu$, then, for any $p$, $q$, any $\Delta_{1}, \Delta_{2}$, and any $f_{1} \in \mathfrak{X}_{p}^{\lambda}\left(\Delta_{1}\right)$ and $f_{2} \in \mathfrak{X}_{q}^{\prime}\left(\Delta_{2}\right)$, the $p$. d. functions $\left(U(g) f_{1}, f_{1}\right)$ and $\left(U(g) f_{2}, f_{2}\right)$ are mutually disjoint. ${ }^{13)}$

13) See $[1], \$ 12$. 
This proposition is evident by Lemma 6 , Proposition 5 and the definition of $\mathfrak{M}_{p}^{\lambda}(\Delta)$.

Proposition 7. Assume that $f_{1} \in \mathfrak{M}_{p}^{\lambda}\left(\Delta_{1}\right)$ and $f_{2} \in \mathfrak{M}_{p}^{\lambda}\left(\Delta_{2}\right)$. In order that the p. d. functions $\left(U(g) f_{1}, f_{1}\right)$ and $\left(U(g) f_{2}, f_{2}\right)$ are not mutually disjoint, it is necessary and sufficient that there exist a Borel set $\Delta \subset \Delta_{1} \cap \Delta_{2}$ such that $\rho(\Delta)$ $>0$ and a B-measurable function $\omega(t)$ defined on $\Delta$ such that $0<|\omega(t)|<\infty$ and that $f_{1}(b, t)=\omega(t) f_{2}(b, t)$ for $\sigma-a . a . b \in \mathbf{K}$ for $\rho-a . a . t \in \Delta$.

Proof. The necessity is clear by Lemma 6.

To prove the sufficiency, we put $\omega_{1}(t)=\min \{1,|\omega(t)|\}$ on $\Delta$ and define

$$
f(b, t)=\left\{\begin{array}{lll}
\omega_{1}(t) f_{1}(b, t) & \text { on } & \mathbf{K} \times \Delta, \\
0 & \text { on } & \mathbf{K} \times(T-\Delta) .
\end{array}\right.
$$

Then we may prove that $f \in \mathfrak{M}_{p}^{\lambda}(\Delta) \subset \mathfrak{M}_{p}^{\lambda}\left(\Delta_{1}\right) \cap \mathfrak{M}_{p}^{\lambda}\left(\Delta_{2}\right)$ and that p. d. function $(U(g) f, f)$ is a common minorant of $\left(U(g) f_{1}, f_{1}\right)$ and $\left(U(g) f_{2}, f_{2}\right)$, q.e.d.

Proposírion 8. In order for $\left\{\mathfrak{M}_{p}^{\lambda}(\Delta), U(g), f\right\}\left(f \equiv f(b, t) \in \mathfrak{M}_{p}^{\lambda}(\Delta)\right)$ to be a cyclic unitary representation of $\mathbf{G}$, it is necessary and sufficient that $f(b, t)$ $\neq 0$ as an element of $\mathfrak{S}_{p}^{\lambda}\left(\subset L^{2}(\mathbf{K})\right)$ for $\rho-a$. $a$. $t \in \Delta$.

Proof. The necessity is clear by the definition of $U(g)$.

We shall prove the sufficiency. Put

$$
\mathfrak{M}^{\prime}=\mathfrak{Q}[\{U(g) f / g \in \mathbf{G}\}]
$$

and let $\varphi$ be any element of $\mathfrak{M}_{p}^{\lambda}(\Delta) \Theta \mathfrak{M}$ '. Then

$$
\int_{\mathbf{K} \times \Delta}\left(\left\langle s_{b}, t\right\rangle, x\right) f(b a, t) \overline{\varphi(b, t)} d b d \rho(t)=0 \quad \text { for any } x \text { and } a .
$$

By the similar argument as in the proof of Lemma 6 , it follows from the above equality that

$$
\int_{\mathbf{K}}\left(\left\langle s_{b}, t\right\rangle, x\right) f(b a, t) \varphi(b, t) d b=0 \quad \text { for any } x \text { and } a
$$

for $\rho \cdots$ a. a. $t \in \Delta$. Since the unitary representation $\left\{\mathscr{g}_{p}^{\lambda}, U_{t}(g)\right\}$ is irreducible for any $t$ (Proposition 1 ) and since $f \neq 0$ in $\mathfrak{S}_{p}^{\lambda}$ for $\rho$-a. a. $t \in \Delta$ by the assumption, we get $\varphi(b, t) \equiv 0$ in $\mathfrak{S}_{p}^{\lambda}$ for $\rho-$ a. a, $t \in \Delta$, and hence $\varphi(b, t) \equiv 0$ in $\mathfrak{M}_{p}^{\lambda}(\Delta)$. Thus we obtain $\mathfrak{M}^{\prime}=\mathfrak{M}_{p}^{\lambda}(\Delta)$, q.e.d.

$\S$ 4. Proof of Theorems. Throughout this paragraph, we notice that the spaec $\mathfrak{M}_{\nu}^{\lambda}$ defined in Theorem 2 is identical with the space $\mathfrak{M}_{1}^{\lambda}\left(\Delta_{2}^{\lambda}\right)$ in the notation stated in $\S 3$ for any $\lambda$ and $\nu$.

Theorems 1.1 and 1.2 have been proved in $\S 2$ - the formula (1.7) may 
be shown by calculating $\mathscr{D}(g) \equiv(U(g) f, f), f=\sum_{\lambda(\alpha, m)=\lambda} \sum_{j} \xi_{j m}^{\alpha} u_{\lambda_{m}+p, j}^{\alpha}$. Theorems 1.4 and 1.5 are evident from the fact $\mathbf{G} / \mathbf{V} \cong \mathbf{K}$ and by Peter-Weyl's theory. (Theorem 1.3 shall be proved after the proof of Theorems 2.1--2.3.)

Next, let $\mathfrak{M}_{\nu}^{\lambda}$ and $f_{\nu}^{\lambda}(\nu=1, \ldots, N(\lambda) ; \lambda=1,2, \ldots)$ be as stated in Theorem 2. Theorem 2.1 have been proved in $\S 3$ (Proposition 4). By the conditions $\left.1^{\circ}\right)$ and $\left.2^{\circ}\right)$, we have $f_{\nu}^{\lambda} \in \mathfrak{M}_{2}^{\lambda}$ and $f_{2}^{\lambda}(b, t) \neq 0$ in $\mathscr{S}_{i}^{\lambda}\left(C L^{2}(\mathbf{K})\right)$ for $\rho-\mathbf{a}$. a. $t \in \Delta_{\nu}^{\lambda}$. Hence the unitary representation $\left\{\mathfrak{M}_{i}^{\lambda}, U(g), f_{\nu}^{\lambda}\right\}$ is cyclic by Proposition 8 for every $\lambda$ and $\nu$. The p. d. functions $\left(U(g) f_{\nu}^{\lambda}, f_{\nu}^{\lambda}\right), \nu=1,2, \ldots$, are mutually disjoint from the condition $3^{\circ}$ ) and by Proposition 7 . Hence, by Theorem 8 in [1], the direct $\operatorname{sum}\left\{\oplus_{\nu} \mathfrak{M}_{\nu}^{\lambda}, U(g), f^{\lambda}\right\}, f^{\lambda}=\sum_{\nu} f_{\nu}^{\lambda}$, is a cyclic unitary representation of G. We may further show by Proposition 6 that the p. d. functions $\left(U(g) f^{\lambda}, f^{\lambda}\right)$ and $\left(U(g) f^{\mu}, f^{\mu}\right)$ are mutually disjoint for $\lambda \neq \mu$. Similar argument is possible for $\left\{\Re_{\nu}^{\alpha}, U(g)\right\}, \nu=1, \ldots, N^{\prime}(\alpha) ; \alpha=1,2, \ldots$. Therefore, by the same argument as in the proof of Theorem 2 in [2], we may prove that the unitary representation $\left\{\xi, U(g), f^{0}\right\}$ stated in Theorem 2.2 is cyclic. The formula (1.12) may be verified by calculating $\Psi(g)=\left(U(g) f^{0}, f^{0}\right)$. Theorem 2.2 is thus proved. Theorem 2.3 may be seen by Proposition 5 .

We now prove Theorem 1.3. If $\left\{\mathscr{S}_{p}^{\lambda}, U_{t_{1}}(g)\right\}$ and $\left\{\mathscr{S}_{q}^{u}, U_{t_{2}}(g)\right\}\left(t_{1} \neq t_{2}\right)$ are mutually unitary equivalent, there exist $f_{1} \in \mathscr{Y}_{j}^{\lambda}$ and $f_{2} \in \tilde{\delta}_{q}^{\lambda}$ such that $\left(U_{t_{1}}(g) f_{1}\right.$, $\left.f_{1}\right)=\left(U_{t_{2}}(g) f_{2}, f_{2}\right)$ for any $g \in \mathrm{G}$, and hence the direct $\operatorname{sum}\left\{\hat{f}_{\dot{p}} \hat{p} \oplus \mathscr{S}_{q}^{u}, U(g), f_{1}\right.$ $\left.+f_{2}\right\}\left(U(g)=U_{t_{1}}(g) \oplus U_{i_{2}}(g)\right)$ is not cyclic by Theorem 8 in [1]. But we may prove by means of Theorems 2.2 and 2.3 verified above that $\left\{\mathscr{S}_{p}^{\lambda} \oplus \mathscr{S}_{q}^{u}, U(g), f_{1}\right.$ $\left.+f_{2}\right\}$ is a cyclic unitary representation of $\mathbf{G}$. That is a contradiction.

In order to prove Theorems 1.6 and 2.4, we first modify Lemma 2 in [2] to the following form:

Lemma 7 . Let $\tilde{X}, S, T$ and $\mathbf{K}$ be as stated in $\S 1$ and $F(1)(1 \subset \widetilde{X} \equiv S \times T)$ be a measure on $\tilde{X}$ such that $F(\tilde{X})<\infty$, and assume that there exists a nonnegative function $u(a ; \%)$ on $\mathbf{K} \times \tilde{X}$, measurable $i n\langle a, \%\rangle$ and summable on $\widetilde{X}$ with respect to $F$ for every $a \in \mathbf{K}$, such that

$$
F(1 a)=\int_{\Delta} u(a ; \%) d F(\%) \quad(A a=\{\% a / \% \in A\})
$$

for any $\Lambda \subset \tilde{X}$ and any $a \in \mathbf{K}$. Then there exist a non-negative B-measurable function $\omega(s, t)$ on $\widetilde{X} \equiv S \times T$ and a measure $\rho(\Delta)$ on $T, \rho(T)<\infty$, such that

$$
F(1)=\int_{\Lambda} \omega(s, t) d s d \rho(t) \text { for any } \Lambda \subset \widetilde{X}
$$

ds being the invariant measure on $S$ defined in $\S 1$.

Proof. We put $B_{\mathrm{A}}=\left\{\langle b, t\rangle /\left\langle s_{b}, t\right\rangle \in \lambda\right\} \subset K \times T$ (see $\S 1$ ) for any $1 \subset \bar{X}$ $\equiv S \times T$, and define a measure $F^{*}(B)$ on $K \times T$ by tho formula: 


$$
\int_{\mathbf{K} \times T} \varphi(b, t) d F^{*}(b, t)=\int_{s \times T} d F(s, t) \int_{\mathbf{K}^{\prime}} \varphi\left(b^{\prime} c_{s}, t\right) d b^{\prime} \quad(\text { see } \S 1)
$$

for any continuous function $\varphi(b, t)$ on $\mathbf{K} \times T$ with compact carrier. Then we have

$$
F^{*}\left(B_{\mathrm{A}}\right)=F(1) \text { for any } \Lambda \subset \tilde{X},
$$

and (4.1) implies

$$
F^{*}(B a)=\int_{B} u^{*}(a ; b, t) d F^{*}(b, t) \quad(B a=\{\langle b a, t\rangle /\langle b, t\rangle \in B\})
$$

where $u^{*}(a ; b, t)=u\left(a ;\left\langle s_{b}, t\right\rangle\right)$ is non-negative, B-measurable in $\langle a, b, t\rangle$ and summable (in $\langle b, t\rangle$ ) on $\mathbf{K} \times T$ with respect to $F^{*}$ for any $a \in \mathbf{K}$. Therefore, by the same argument as the proof of Lemma 2 in [2], we may show that there exist a non-negative B-measurable function $\omega^{*}(s, t)$ on $\mathbf{K} \times T$ and a measure $\rho$ on $T, \rho(T)<\infty$, such that

$$
F^{*}(B)=\int_{3} \omega *(b, t) d b d \rho(t) \text { for any } B \subset \mathbf{K} \times T .
$$

Hence we obtain from $(4.4),(1.2)$ and by simple calculation that

$$
F(A)=\int_{\Lambda} d s d \rho(t) \int_{\mathbf{K}^{\prime}} \omega^{*}\left(b^{\prime} c_{s}, t\right) d b^{\prime} \quad \text { for any } \quad A \subset \tilde{X}
$$

and hence we get $(4.2)$ by putting $\omega(s, t)=\int_{\mathbf{K}^{\prime}} \omega^{*}\left(b^{\prime} c_{s}, t\right) d b^{\prime}$, q.e.d.

Hereafter the indices $j$ and $k$ may run over all natural numbers, not following after the rule defined in $\S 1$.

Now let $\left\{\mathscr{g}, U(g), f^{0}\right\}$ be a cyclic unitary representation of $\mathbf{G}$. Then, making use of Lemma 7, we can achieve the same argument as in [2] - from the $\mathrm{b} \in$ ginning of $\$ 3(\mathrm{p} .6)$ to L. 14 in p. $10-$, and obtain the following result:

$\{\mathfrak{g}, U(g)\}=\{\Re, U(g)\} \oplus\{\mathfrak{M}, U(g)\} ;\{\Re, U(g)\}$ is equivalent to a cyclic unitary representation of the group $\mathbf{K}(\cong \mathbf{G}, \mathbf{V})$, and $\{\mathfrak{M}, U(g)\}$ is given as follows: there exists a unitary space $\hbar_{0}$ of all sequences of complex numbers: $\left\{\hat{\xi}_{1}, \ldots\right.$, $\left.\xi_{n}\right\}, n \leqq \infty$, such that $\|\xi\|^{2}=\sum_{j=1}^{\infty} \mid \xi_{j i}{ }^{2}<\infty \quad($ if $n=\infty)$, and exists a matrix of functions $M(a ; s, t)=\left\|u_{j k}(a ; s, t)\right\|$ whose elements $u_{j k}(a ; s, t)(j, k=1, \ldots, n)$ are $\mathrm{B}$-measurable in $\langle a, s, t\rangle$; and every $f \in \mathfrak{M}$ is realized as a $\tilde{D}_{0}$-valued function $\mathbf{f}(s, t) \equiv\left\{f_{1}(s, t), \ldots, f_{n}(s, t)\right\}$ defined on $\widetilde{X} \equiv S \times T$, and $f \sim \mathbf{f}(s, t)^{14)} \mathrm{im}$ plies that

$$
\left\{\begin{array}{l}
\|f\|^{2}=\int_{s \times T}\|\mathbf{f}(s, t)\|^{2} d s d \rho(t) \quad\left(\|\mathbf{f}(s, t)\|^{2}=\sum_{j}\left|f_{j}(s, t)\right|^{2}\right) \\
U(x) f \sim(\langle s, t\rangle, x) \mathbf{f}(s, t) \quad \text { for any } \quad x \in \mathbf{V} \\
U(a) f \sim M(a ; s, t) \mathbf{f}(s a, t) \quad \text { for any } \quad a \in \mathbf{K}
\end{array}\right.
$$

14) $f \sim f(s, t)$ means that $f$ is realized as $\mathbf{f}(s, t)$. 
$\rho$ being a measure on $T$ such that $\rho(T)<\infty$ (obtaind from Lemma 7).

Next, for any B-measurable function $f(s, t)$ on $S \times T$, we define a function $f^{*}(b, t)$ on $\mathbf{K} \times T$ by

$$
f^{*}(b, t) \equiv f(s b, t)
$$

and put $M^{*}(a ; b, t) \equiv\left\|u_{j k}^{*}(a ; b, t)\right\|$. Then, as is easily seen, the above result concerning $\{\mathfrak{M}, U(g)\}$ is translated into the following form: every $f \in \mathfrak{M}$ is realized as a $\$_{2}$-valued function $\mathbf{f}(b, t)$ defined on $\mathbf{K} \times T$ and $f \sim \mathbf{f}(b, t)$ implies that

$$
\left\{\begin{array}{l}
\|f\|^{2}=\int_{\mathbf{K} \times T}\|\mathbf{f}(b, t)\|^{2} d b d \rho(t), \\
U(x) f \sim(\langle s b, t\rangle, x) \mathbf{f}(b, t) \text { for any } x \in \mathbf{V}, \\
U(a) f \sim M^{*}(a ; b, t) \mathbf{f}(b a, t) \text { for any } a \in \mathbf{K} ;
\end{array}\right.
$$

moreover, if $M_{1}^{*}(a ; b, t)=M_{2}^{*}(a ; b, t)$ as operators in $\mathfrak{M}$, then $M_{1}^{*}(a ; b c, t)$ $=M_{2}^{*}(a ; b c, t)$ in the same sense for any $c \in \mathbf{K}$ - see p. 10 in [2].

Starting from this result, we can achieve the similar argument to that in [2] - from p. 10 , L. 15 to p. 11 , L. $15 .^{15}$ ) Thus $\mathfrak{M}$ may be realized as a subspace of the direct sum of at most countable number of $L^{2}(\mathbf{K} \times T, \sigma \otimes \rho)$, and $f \sim\left\{\psi_{\nu}(b, t)\right\} \equiv\left\{\psi_{1}(b, t), \psi_{2}(b, t), \ldots\right\}$ implies

$$
\begin{cases}\|f\|^{2}=\sum_{\nu=1}^{n} \int_{\mathbf{K} \times T}\left|\psi_{\nu}(b, t)\right|^{2} d b d \rho(t), & n \leqq \infty, \\ U(x) f \sim\left\{\left(\left\langle s_{b}, t\right\rangle, x\right) \psi_{\nu}(b, t)\right\} & \text { for any } x \in \mathbf{V}, \\ U(a) f \sim\left\{\psi_{\nu}(b a, t)\right\} \text { for any } a \in \mathbf{K} .\end{cases}
$$

Since $L^{2}(\mathbf{K} \times T, \sigma \otimes \rho)=\bigoplus_{\lambda=1}^{\infty} \bigoplus_{p=1}^{\tilde{n}(\lambda)} \mathfrak{M}_{p}^{\lambda}(T)$ by Lemma 5 and Proposition 4 (§3), it follows that $\mathfrak{M}$ may be expressible in the form:

$$
\mathfrak{M}=\bigoplus_{\lambda=1}^{\infty} \bigoplus_{p=1}^{\tilde{n}(\lambda)} \bigoplus_{\nu=1}^{n(\lambda, p)} \mathfrak{M}_{\nu p}^{\lambda} \quad(n(\lambda, p) \leqq \infty), \quad \mathfrak{M}_{\nu p}^{\lambda} \subset \mathfrak{M}_{p}^{\lambda}(T) \quad \text { for any } \quad \nu,
$$

and every $\mathfrak{M}_{2, \phi}^{\lambda}$ is a closed linear subspace of $\mathfrak{M}$ invariant under $U(g), g \in \mathbf{G}$.

Put

$$
f^{0}=f+h, \quad f \in \mathfrak{M} \text { and } h \in \mathfrak{R},
$$

and

$$
f=\sum_{\lambda} \sum_{p} \sum_{\nu} f_{\nu p}^{\lambda}, \quad f_{\nu p}^{\lambda} \in \mathfrak{M}_{\nu p}^{\lambda} \quad\left(\subset \mathfrak{M}_{p}^{\lambda}(T)\right) .
$$

Then $\{\mathfrak{M}, U(g), f\}$ is - and consequently every $\left\{\mathfrak{M}_{\nu p}^{\lambda}, U(g), f_{\nu p}^{\lambda}\right\}$ is a cyclic unitary representation of $\mathbf{G}$. We put

ii) Such argument is impossible without extending functions on $S \times T$ to those on $K \times T$ as stated above. The author owes to Mr. S. Murakami's suggestion for this improvement. 


$$
\Delta_{\nu p}^{\lambda}=\left\{t / \int_{\mathbf{K}}\left|f_{i p}^{\lambda}(b, t)\right|^{2} d b \neq 0\right\}(\subset T) .
$$

Then $\left\{\mathfrak{M}_{\nu p}^{\lambda}, U(g), f_{\nu p}^{\lambda}\right\}$ is cyclic if and only if $\mathfrak{M}_{\nu p}^{\lambda}=\mathfrak{M}_{p}^{\lambda}\left(\Delta_{\nu p}^{\lambda}\right)$ by Proposition 8 . We may consider by Proposition 5 that $\mathfrak{M}_{\nu p}^{\lambda}=\mathfrak{M}_{1}^{\lambda}\left(\Delta_{\nu p}^{\lambda}\right)$ and $f \in \mathfrak{M}_{1}^{\lambda}\left(\Delta_{\nu p}^{\lambda}\right)$. Exchanging indices, we denote for any $\lambda$

$$
\Delta_{\nu}^{\lambda} \text { and } f_{\nu}^{\lambda}, \quad \nu=1, \ldots, N(\lambda)(\leqq \infty)
$$

instead of

$$
\begin{aligned}
& \Delta_{\nu p}^{\lambda} \text { and } f_{\nu p}^{\lambda}, \\
& \nu=1, \ldots, n(\lambda, p)(\leqq \infty) ; p=1, \ldots, \tilde{n}(\lambda)(<\infty) ;
\end{aligned}
$$

and put $\mathfrak{R}_{\nu}^{\lambda}=\mathfrak{M}_{1}^{\lambda}\left(\Delta_{\nu}^{\lambda}\right)$. Then we may consider that

$$
\{\mathfrak{M}, U(g)\}=\bigoplus_{\lambda=1}^{\infty} \bigoplus_{\nu=1}^{N(\lambda)}\left\{\mathfrak{M}_{\nu}^{\lambda}, U(g)\right\}, \quad f=\sum_{\lambda} \sum_{\nu} f_{\nu}^{\lambda},
$$

and

$$
f_{\nu}^{\lambda} \in \mathbb{M}_{1}^{\lambda}\left(\Delta_{\nu}^{\lambda}\right), \quad f_{\nu}^{\lambda}(b, t) \neq 0 \text { in } \mathfrak{D}_{1}^{\lambda} \text { for } \rho-\text { a. a. } t \in \Delta_{\nu}^{\lambda} \text {. }
$$

Hence

$$
f_{:}^{\lambda}(b, t)=\sum_{\lambda(\alpha, m)=\lambda} \sum_{j} u_{\nu_{m}+1, j}^{\alpha}(b) f_{\nu j}^{\alpha m}(t) \quad \text { (convergence in } L^{2}(\mathbf{K} \times T, \sigma \otimes) \text { ) }
$$

for any $\lambda$ and $\nu$ where the series of functions

$$
\left\{\left.f_{\nu j}^{\alpha m}\right|_{\nu=1, \ldots, N(\lambda) ; \lambda=1,2, \ldots} ^{j=1, \ldots, n(\lambda) ; \lambda(\alpha, m)=\lambda ;}\right\}
$$

satisfies the conditions $\left.1^{\circ}\right)$ and $\left.2^{\circ}\right)$ in Theorem 2.2. Since $\{\mathfrak{R}, U(g), f\}$ is cyclic, it follows from (4.5) and by Theorem 8 in [1] that p. d. functions $\left(U(g) f_{\nu}^{\lambda}\right.$, $\left.f_{\nu}^{\lambda}\right), \nu=1, \ldots, N(\lambda), \lambda=1,2, \ldots$, are mutually disjoint. Ifence the series $\left\{f_{i j}^{\alpha m}\right\}$ satisfies the condition $3^{\circ}$ ) by Propositions 6 and 7. Therefore $\{\mathfrak{M}, U(g)$, f) must be of form stated in Theorem 2.2. Similar argument may be achieved for $\{\mathfrak{H}, U(g), h\}$. Consequently we obtain (1.10), (1.11) and (1.12) by simple calculations. Theorem 2.4 is thus proved.

Next, assume that the cyclic unitary representation $\left\{\mathscr{g}, U(g), f^{0}\right\}$ is irreducible. (Notice that any irreducible representation is cyclic.) Then only one couple $\langle\lambda, \nu\rangle$ or $\langle\alpha, \nu\rangle$ may be appear in (1.10). In the case $\{\mathfrak{g}, U(g)\}=\left\{\mathfrak{M}_{\nu}^{\lambda}\right.$, $U(g)\}$, by the irreducibility, there exists a point $t_{0} \in T$ such that $\rho\left(T-\left\{t_{0}\right\}\right)=0$. Hence $\{\mathfrak{F}, U(g)\}$ must be of the form stated in Theorem 1.1 or 1.4. Thus we obtain Theorem 1.6.

Finally, Theorem 3 is easily seen from Theorems 1 and 2 .

\section{LITERATURE}

[1] R. Godement: Les fonctions de type positif et la theorie des groupes, Trans. Amer. 
Math. Soc. 63, No. 1 (1948) pp. 1-84.

[2] S. Itò: Unitary representations of some linear groups: Nagoya Math. Journ. 4 (1952) pp. 1-13.

[3] G. W. Mackey: Imprimitivity for representations of locally compact groups I, Proc. Nat. Acad. Sci. 35, No. 9 (1949) pp. 537-543.

[4] G. W. Mackey: On induced representations of groups, Amer. J. Math. 73, No. 3 (1951) pp. 576-592.

[5] G. W. Mackey : Induced representations of locally compact groups I, Ann. of Math. 55, No. 1 (1952) pp. 101-139.

[6] A. Weil: L'intégration dans les groupes topologiques et ses applications, Act. Sci. Ind. Paris, 869 (1940).

Mathematical Institute, Nagoya University 